\title{
Acknowledgement to Reviewers of Actuators in 2017
}

\author{
Actuators Editorial Office \\ MDPI AG, St. Alban-Anlage 66, 4052 Basel, Switzerland
}

Published: 15 January 2017

Peer review is an essential part in the publication process, ensuring that Actuators maintains high quality standards for its published papers. In 2017, a total of 31 papers were published in the journal. Thanks to the cooperation of our reviewers, the median time to first decision was 27 days and the median time to publication was 71 days. The editors would like to express their sincere gratitude to the following reviewers for their time and dedication in 2017:

Abidi, Khalid

Cao, Xin

Chasalevris, Athanasios

Cheong, Joono

Coskun, Bulut

Daerden, Frank

Dagdeviren, Canan

Debéda, Hélène

Deng, Mingcong

Di, Long

Dubois, Fabien

Eckert, Paulo Roberto

Felton, Samuel

$\mathrm{Fu}, \mathrm{Xin}$

Gaviria-López, Carlos Alberto

Groothuis, Stefan

$\mathrm{Gu}$, Guoying

Han, Bangcheng

Hatzfeld, Christian

Herold, Sven

Hofer, Matthias

Hondori, Hossein

Hošovský, Alexander

Hunstig, Matthias

Hur, Deog Jae

Jang, Sung-Hwan

Jastrzebski, Rafal Piotr

Jiang, Dong

Jugo, Josu

Kaal, William

Kadumudi, Firoz Babu

Kanda, Takefumi

Kermani, Mehrdad R.

Kiefer, Rudolf

Kim, Yeongjin

Knospe, Carl
Komori, Mochimitsu

Kong, Ling Bing

Kouh, Taejoon

LaHaye, Matthew

Lee, Jae Shin

Lee, Yong Bok

Li, Shiyang

Liu, Shuqin

Liu, Tzong-shi

Maas, Jürgen

Merola, Alessio

Misgeld, Berno

Moriello, Lorenzo

Müller, Bert

Murrenhoff, Hubertus

Mystkowski, Arkadiusz

Naso, David

Nurmi, Jarmo

Oldham, Kenn

Pantelis, Nikolakopoulos

Pesch, Alexander $\mathrm{H}$.

Pitel', Ján

Plooij, Michiel

Pott, Peter

Pręgowska, Agnieszka

Rabenorosoa, Kanty

Rakotondrabe, Micky

Rauch, Erwin

Ristic, Michael

Rosendo, Andre

Saadeh, Mohammad

Schneider, Ben $\mathrm{H}$.

Serpilli, Michele

Shiao, Yaojung

Siqueira, Adriano

Skidmore, Jeffrey 
Stanley, Andrew A.

$\mathrm{Su}$, Chun-yi

Suzumori, Koichi

Tadrist, Loïc

Takosoglu, Jakub Emanuel

Tarisciotti, Luca

Vanderborght, Bram
Wang, Shiqian

$\mathrm{Wu}$, Jiagang

Yeow, Chen Hua

Zhan, Haifei

Zhang, Dou

Zhao, Huichan

Zhou, Jin

(c)

(C) 2018 by the author. Licensee MDPI, Basel, Switzerland. This article is an open access article distributed under the terms and conditions of the Creative Commons Attribution (CC BY) license (http://creativecommons.org/licenses/by/4.0/). 